\title{
REPRESENTAÇÕES SOCIAIS DE GESTANTES QUE FREQUENTAM SERVIÇO ESPECIALIZADO EM GESTAÇÕES DE ALTO RISCO
}

\author{
SOCIAL REPRESENTATIONS OF PREGNANT WOMEN ATTENDING SPECIALIZED SERVICE IN HIGH RISK \\ MANAGEMENT
}

\section{REPRESENTACIONES SOCIALES DE MUJERES EMBARAZADAS QUE ASISTEN A UN SERVICIO ESPECIALIZADO EN GESTIÓN DE ALTO RIESGO}

Sayonara Natália Ferreira ${ }^{1}$, Marina Pereira Lemos ${ }^{2}$, Walquíria Jesusmara Santos ${ }^{3}$

\section{RESUMO}

Objetivo: compreender as representações sociais e experiências das gestantes que frequentam serviços especializados em gestação de alto risco. Método: estudo qualitativo, realizado com mulheres em assistência pré-natal de um serviço público especializado em Gestação de Cuidados Especiais de um município mineiro de médio porte. A coleta de dados ocorreu, por meio de entrevista semiestruturada na qual se utilizou as noções da Teoria das Representações Sociais e os dados organizados, por meio da Análise Estrutural de Narração, para realizar a interpretação das respostas. Resultado: as representações encontradas estão em torno das ideias relacionadas ao risco presente nas gestações e à experiência vivenciada nos atendimentos realizados nos serviços de referência, emergindo da análise três categorias temáticas: representações relacionadas ao modelo biomédico, representações relacionadas ao medo e representações relacionadas à morte. Conclusão: como consequência desse estudo, foi possível evidenciar que as representações das gestantes de alto risco giraram em torno de sentimentos de medo relacionado à gestação e da morte em decorrência dos agravos da condição de risco, além da valorização do modelo biomédico e do profissional de medicina em detrimento do trabalho em equipe multiprofissional e da promoção da saúde.

Descritores: Cuidado Pré-natal; Enfermagem; Gestação de Alto Risco.

\begin{abstract}
Objective: to understand the social representations and experiences of pregnant women who attend specialized services in high-risk pregnancies. Method: qualitative study carried out with women in prenatal care from a public service specializing in Special Care Gestation in a medium-sized town in Minas Gerais, Brazil. Data collection through a semi-structured interview in which the notions of the Theory of Social Representations and the data organized through the Structural Narration Analysis were used to interpret the responses. Result: the representations are around the ideas related to the risk present in pregnancies and the experience lived in the care provided in reference services, emerging from the analysis three thematic categories: representations related to the biomedical model, representations related to fear and representations related to death. Conclusion: as a result of this study, it was possible to show that the representations of high-risk pregnant women revolved around feelings of fear related to pregnancy and death due to the worsening of the risk condition, in addition to the valorization of the biomedical model and the health professionals in detriment of multiprofessional teamwork and health promotion.
\end{abstract}

Descriptors: Prenatal Care; Nursing; Pregnancy, High-Risk.

\section{RESUMEN}

Objetivo: comprender las representaciones sociales y las experiencias de las mujeres embarazadas que asisten a servicios especializados en embarazos de alto riesgo. Método: estudio cualitativo, realizado con mujeres en atención prenatal de un servicio público especializado en gestación de atención especial en una ciudad mediana de Minas Gerais. La recopilación de datos se realizó a través de una entrevista semiestructurada en la que se utilizaron las nociones de la Teoría de las representaciones sociales y los datos organizados a través del Análisis estructural de la narración para interpretar las respuestas. Resultado: las representaciones encontradas giran en torno a las ideas relacionadas con el riesgo presente en los embarazos y la experiencia vivida en la atención prestada en los servicios de referencia, emergiendo del análisis tres categorías temáticas: representaciones relacionadas con el modelo biomédico, representaciones relacionadas con el miedo y representaciones relacionadas con la muerte. Conclusión: Como resultado de este estudio, fue posible mostrar que las representaciones de mujeres embarazadas de alto riesgo giraban en torno a sentimientos de miedo relacionados con el embarazo y la muerte debido al empeoramiento de la condición de riesgo, además de la valorización del modelo biomédico y el profesional de la salud. medicina en detrimento del trabajo en equipo multiprofesional y la promoción de la salud.

Descriptores: Atención prenatal; Enfermería; Embarazo de alto riesgo.

${ }^{1}$ Acadêmica de Enfermagem pela Universidade Federal de São João Del Rei. ${ }^{2}$ Acadêmica de Enfermagem pela Universidade Federal de São João Del Rei. ${ }^{3}$ Doutora em Enfermagem pela Universidade Federal de Minas Gerais. Docente do Curso de Enfermagem da Universidade Federal de São João Del Rei.

Ferreira SN, Lemos MP, Santos WJ. Representações Sociais de que frequentam serviço especializado em gestações de alto Risco. Revista de Enfermagem do Centro Oeste Mineiro. 2020;10:e3625. [Access__]. Available in:___. DOI: http//doi.org/10.19175/recom.v10i0.3625 


\section{INTRODUÇÃO}

O período gestacional da mulher tem duração média de 40 semanas. Nesse período, a gestante redescobre seu corpo, de acordo com as mudanças que acontecem para que o organismo se adapte, para promover o desenvolvimento do bebê. Dessa forma, é comum que essa etapa gere contradições entre as experiências de cada gestante em consequência das alterações fisiológicas, emocionais e comportamentais que cada mulher vive nesse processo ${ }^{(1)}$.

Diante disso, o Ministério da Saúde, por meio da Rede Cegonha, instituiu, em 2011, as diretrizes para a melhoria da atenção às gestantes no pré-natal, parto, puerpério e na atenção à saúde da criança, além da regulação de leitos obstétricos e neonatais e transporte seguro nas situações de alto risco, suprindo todas as demandas das mulheres e permitindo que elas tenham uma experiência positiva sobre a gestação. Dentre essas diretrizes propostas na atenção pré-natal, ressaltam-se ações de cuidado e promoção em saúde, rastreio diagnóstico e prevenção de doenças e melhoria da comunicação entre gestantes e os serviços de saúde ${ }^{(2)}$.

No Brasil, percebe-se uma melhora no atendimento pré-natal oferecido pelo Sistema Único de Saúde (SUS), com a redução nas taxas de mortalidade materno-infantil, por meio da implantação e implementação de programas e políticas públicas voltadas para garantir a cobertura da assistência pré-natal. As mortes maternas, por causas obstétricas, podem ser de dois tipos: causas diretas (intercorrências mal abordadas, durante o período gestacional, parto e pós-parto) e causas indiretas (os óbitos ligados às doenças já existentes ou que surgem durante a gestação) sendo possível a redução ou até a descontinuidade das ocorrências desse grupo, uma vez que receberem atenção adequada ${ }^{(3)}$.

Com as mudanças instituídas, a partir da Rede Cegonha, o país mostrou redução nos índices de mortalidade materno-infantil e, a despeito das políticas, dos esforços do governo e das entidades de classes envolvidas com essa situação, a projeção da razão de mortalidade materna para 2030 é de 30 óbitos por 100 mil nascidos vivos ${ }^{(1)}$. Para tal, é importante que a Unidade Básica de Saúde (UBS) seja a porta de entrada da gestante, onde ela receberá atenção voltada às suas necessidades, garantindo, assim, acompanhamento contínuo e de qualidade dessa gestação ${ }^{(3)}$.
A partir das medidas já estabelecidas, a UBS inicia a assistência ao reconhecer a existência da gestação, então realiza a primeira consulta prénatal com caráter exploratório, buscando informações e realizando exames para fazer a classificação de risco de acordo com os dados obtidos. Essas ações respondem ao principal objetivo da assistência pré-natal que é o acolhimento e a adesão da gestante para assegurar que a gestação transcorra de forma saudável para o binômio mãe-filho ${ }^{(4)}$. A partir de então é oferecido um acompanhamento que se adéque a cada caso.

Em casos onde doenças ou possíveis riscos sejam observados a gestante é referenciada ao Serviço de Referência em Pré-natal de Cuidados Especiais ou Alto Risco de cada região, recebendo atenção voltada para suas necessidades, sem, portanto, perder o vínculo com a UBS mais próxima de sua residência ${ }^{(1)}$.

Estudo voltado para as percepções e vivências de mulheres diagnosticadas com algum risco gestacional, aponta que a vivência de agravos, nesse período, pode fazer com que essas gestantes se sintam mais vulneráveis, podendo atuar de forma desfavorável na saúde da gestante e do concepto. Além do mais, o enfrentamento de uma situação negativa relacionada à sua saúde ou do bebê pode gerar sofrimento, o que, direta ou indiretamente pode influenciar no autocuidado e no cuidado pré-natal ${ }^{(5)}$.

Assim, é de extrema importância que os profissionais de saúde conheçam as representações sociais e percepções das gestantes diagnosticadas com algum agravo ou situação de risco gestacional, para que possam desenvolver ações voltadas para o empoderamento e autocuidado, visto que, a comunicação efetiva proporciona maior confiança na relação entre a gestante e os profissionais de saúde além de possibilitar o reconhecimento das demandas e inquietações da mulher, nessa fase da vida ${ }^{(6)}$. $\mathrm{Na}$ equipe de saúde destacam-se os profissionais de enfermagem, especialmente o enfermeiro, como referência no cuidado às gestantes de alto-risco, no desenvolvimento de ações preventivas e promocionais de saúde, assumindo um papel fundamental na promoção de boas práticas e na elucidação de conhecimentos a respeito da gravidez, parto e pós-parto, por possuir formação técnica, científica e humanística, e as condutas adotadas por esse profissional na atenção prénatal são diretamente proporcionais à qualidade da assistência prestada ${ }^{(6-7)}$. 
Nas gestações que necessitam de cuidados especiais, o próprio fator de risco configura-se um componente estressante, que pode levar à ansiedade e dificuldade de adaptação emocional. Além do mais, as expectativas com relação à evolução da gestação, parto e cuidados com o recém-nascido e o vínculo mãe-bebê podem ser influenciadas pelas crenças e experiências dessas mulheres, durante o período gestacional. Nesse contexto, torna-se extremamente importante conhecer as crenças e representações das gestantes sobre suas condições de risco, de modo a ajuda-las a elaborar mais adequadamente suas emoções, incentivar o enfrentamento e desmistificar suas crenças. Desse modo, o objetivo desse estudo é compreender as representações sociais e experiências das gestantes que frequentam serviços especializados em gestação de alto risco. Diante do exposto, questiona-se: quais são as representações sociais de gestantes que apresentam risco gestacional e como o diagnóstico de risco afeta a vida das mulheres no âmbito social e emocional?

\section{MÉTODO}

Trata-se de um estudo qualitativo fundamentado no referencial teórico das Representações Sociais. A Teoria das Representações Sociais (TRS) foi embasada nas ideias de Moscovici que sugeriu a existência de um pensamento social decorrente das crenças e experiências originadas na vida cotidiana, no curso das comunicações interpessoais que funciona como uma espécie de teoria do senso comum. A TRS tem sido muito utilizada nos estudos na área de saúde e tem contribuído na definição epistemológica moscoviciana, para a reconfiguração dos objetos de pesquisa, de intervenção e também de práticas de saúde. A escolha desse referencial se deu, porque a construção das representações permite que os grupos identifiquem suas necessidades humanas e necessidades de saúde e, principalmente, a ressignificação dessas necessidades ${ }^{(8)}$.

O cenário de estudo foi um Serviço de Referência Especializado em Pré-natal de Alto Risco de um município de médio porte de Minas Gerais. A escolha dessa instituição se justificou, por ser um serviço de referência, na região em que está inserida com suas atividades voltadas ao tratamento de riscos e agravos diagnosticados na gestação e prevenção de complicações. O Serviço funciona em uma unidade que atende a outros grupos populacionais, do tipo Centro de
Especialidades Médicas e a equipe de enfermagem e multidisciplinar, com exceção dos médicos especialistas atendem a usuários do serviço como um todo. Não faz parte da rotina da unidade à realização de consultas de enfermagem às gestantes de alto-risco e outros profissionais da equipe multidisciplinar atendem a essa população de forma agendada de acordo com encaminhamentos. A coleta de dados foi realizada, entre janeiro e setembro de 2019 , e o projeto foi aprovado pelo Comitê de Ética em Pesquisa da Universidade Federal de São João DelRei, Campus Centro-Oeste (CEPCO) sob o Parecer no 3.173.674.

Para tanto, participaram do estudo 17 mulheres que atenderam aos seguintes critérios de inclusão: estar grávida e ter recebido diagnóstico de algum risco gestacional, na atual gestação, ter idade igual ou superior a 18 anos, terem plena capacidade de ação, aceitarem participar do estudo e estar fora de crise e em condições de diálogo, em caso de transtorno mental ou uso de drogas. Os dados foram coletados, utilizando-se o critério de saturação, para definir a interrupção da coleta ou a inclusão de novas entrevistas, ou seja, quando se detecta a reincidência de informações, sem deixar de se considerar, entretanto, informações ímpares levadas em conta na busca da essência do fenômeno em cada uma das entrevistas ${ }^{(9)}$. Para contatá-las, as pesquisadoras compareceram ao serviço, em dias previamente acordados com a gerência e o convite foi realizado, antes ou logo após o atendimento dos profissionais de saúde da instituição. Houve a apresentação formal da pesquisa explicando os critérios éticos e direitos legais das participantes.

Como fonte de evidências, foi utilizada a entrevista semiestruturada e, inicialmente, foram realizadas perguntas referentes às condições sociodemográficas seguidas das seguintes questões norteadoras: "Conte-me, o que você pensa sobre gravidez de risco.", "Como você se sentiu ao receber a notícia que seria encaminhada para um serviço de Pré-natal de Alto Risco?", "Atualmente, como você se sente com relação à gravidez?", "Conte-me sobre o seu atendimento, aqui no serviço de Pré-natal de Alto Risco." As entrevistas foram gravadas em dispositivo digital e, posteriormente, transcritas na íntegra. Para preservar o sigilo, a identificação das participantes se deu, por meio da adoção da letra $E$ (Entrevistada), seguido de número sequencial às entrevistas realizadas aleatoriamente (E1, E2, E3, 
E4...) e a divulgação dos resultados foi feita de forma a não identificar as voluntárias.

Os dados foram tratados, por meio da análise estrutural da narração descrita por Demazière e Dubar ${ }^{(10)}$. Na análise estrutural da narração, as entrevistas dos sujeitos de pesquisa são consideradas como uma reflexão dos temas abordados. Os relatos das entrevistadas são tratados, a priori, e incondicionalmente, como uma verdade, levando em consideração que a narrativa corresponde àquilo em que acreditam, sendo, então, posteriormente, interpretados. Ao pesquisador, cabe tratar as entrevistas em sua totalidade, abrangendo as contradições, conjunções e possíveis disjunções das falas ${ }^{(10)}$.

A análise estrutural da narração é constituída por três etapas descritas por Blanchet e Gottman ${ }^{(11)}$ : a leitura vertical, a horizontal e a transversal. Na leitura vertical, buscamos extrair o sentido global de cada entrevista, permitindo o levantamento dos temas presentes. Durante a leitura horizontal, realizamos a desconstrução e reconstrução de cada entrevista, o que nos permitiu explicitar as significações atribuídas pelos sujeitos entrevistados aos objetos citados nas narrativas. As entrevistas foram sequenciadas (S) por temas/enunciados,, enumeradas de forma crescente, por ordem de aparecimento nas narrativas. Em cada sequência identificados os fatos narrados (F), as justificativas, explicações e reflexões sobre esses fatos (J), e os participantes da pesquisa, ou seja, pessoas que fizeram parte da pesquisa $(P)$. Posteriormente, as sequências foram agrupadas por assunto tratado, sendo realizada uma categorização dos dados. Na leitura transversal, comparamos e destacamos os significados surgidos nas entrevistas, naquilo em que foram concordantes e discordantes. A última etapa do trabalho consistiu em levantar categorias de base do estudo, construídas a partir do referencial teórico, para comparação e discussão dos resultados com outros estudos.

\section{RESULTADOS E DISCUSSÕES}

Dentre as participantes da pesquisa, dezesseis foram encaminhadas pela unidade básica de saúde e uma foi encaminhada pela maternidade de referência, em razão de uma intercorrência no primeiro trimestre. A faixa etária das participantes estava entre 23 e 41 anos, sendo a média de idade 32 anos. A renda familiar de cinco dessas mulheres está entre dois e quatro salários mínimos, uma delas está acima de cinco salários mínimos e as demais relataram renda de até dois salários mínimos. Entre as condições de risco apontadas pelas participantes 5,8\% apresentaram doença hipertensiva da gravidez; $35,2 \%$ hipertensão arterial crônica (HAC); $11,76 \%$ diabetes mellitus gestacional; 5,8\% HAC + DM gestacional; $17,6 \%$ doenças autoimunes; $5,8 \%$ mioma uterino; $5,8 \%$ descolamento prévio da placenta e $11,7 \%$ não souberam especificar. Com relação à história gestacional, quatro são nulíparas e treze multíparas, sendo que seis entrevistadas relataram abortamentos em gestações anteriores. A idade gestacional das participantes, no momento da entrevista, variou entre seis e trinta e nove semanas.

As representações encontradas estão em torno das ideias relacionadas ao risco presente nas gestações e à experiência vivenciada nos atendimentos realizados nos serviços de referência, emergindo da análise três categorias temáticas: representações relacionadas ao modelo biomédico, representações relacionadas ao medo e representações relacionadas à morte.

\section{Representações relacionadas ao modelo biomédico}

$\mathrm{Na}$ primeira categoria temática, as representações das mulheres entrevistadas revolveram em torno da valorização do modelo biomédico, do atendimento médico-centrado e da estrutura do serviço de referência especializado.

A partir das falas, percebe-se a valorização do atendimento centrado no profissional médico e que o conhecimento e a prática em saúde são centralizados somente nesse profissional, sendo evidente a ausência do reconhecimento da assistência de outros atores. "A gente sente que está sendo acompanhada por um bom médico que já está acostumado com tudo isso" (E7). "As duas vezes que fiz o pré-natal aqui, eu fiz com o doutor $X$, os outros médicos (e profissionais) que atendem aqui eu não conheço" (E8). $O$ estudo de Amorin et al. ${ }^{(12)}$ aponta que, apesar das políticas públicas de atenção à saúde da mulher, nos âmbitos da gestação de risco, avançarem na prestação de serviço assistencial, esta evolui no ponto de vista do modelo biomédico e que se faz necessário considerar que os resultados alcançados com uma equipe multiprofissional, em que se insere a enfermagem, pode oferecer, além de atendimento clínico, suporte emocional e educativo em todos os momentos de cuidado.

Sobre o serviço especializado, as falas demonstram que a qualidade e resolutividade do serviço eram traduzidas em realização de exames 
e pela continuidade do acompanhamento pelo mesmo profissional: [...] O que eu achei legal em relação aos médicos é que é sempre a mesma médica que me atende, desde a minha primeira consulta aqui" (E1). "Ele é um médico que eu confio e tudo que eu pergunto ele me esclareceu da melhor forma possível, sempre me solicitou todos os exames. $\mathrm{O}$ acolhimento que a gente tem aqui e a estrutura já dá uma calma. Então, eu me sinto mais segura de fazer o pré-natal aqui, porque o acompanhamento médico é diferenciado" (E8).

Outro estudo aponta que a centralidade do pré-natal de alto-risco reside em consultas médicas sem estabelecer interface com questões socioeconômicas, os quais sabidamente interferem na saúde materno-infantil ${ }^{(13)}$. De acordo com Cardelli et al. ${ }^{(14)}$, corroborando os resultados desse estudo, o cuidado pré-natal é um momento essencial para uma gravidez segura, embora seja, muitas vezes, centrado na figura do médico e na garantia de acesso a exames laboratoriais e de imagem.

Nesse sentido, a consulta de enfermagem no pré-natal permite identificar problemas e necessidades das gestantes, elaborar o planejamento de ações e cuidados necessários e aplicar o processo de enfermagem como instrumento metodológico que sistematiza a consulta e fundamenta a tomada de decisões ${ }^{(15)}$. Entretanto, observa-se nos serviços de atenção secundária, uma lógica voltada para o atendimento médico com pouca participação de outros profissionais, incluindo o enfermeiro, o que foi observado também neste estudo. Apesar de o serviço contar com uma equipe multiprofissional, há uma predominância do modelo biomédico, traduzido em consultas médicas e realização de exames complementares. A atuação do profissional enfermeiro como parte integrante da assistência às gestantes de alto-risco pode superar a visão preestabelecida, quando, então, o enfermeiro tem atuação limitada em espaços onde há o predomínio de tecnologias dura e levedura. Assim, os Serviços de Atenção ao Pré-Natal de Alto-Risco se configuram em espaços onde o enfermeiro possa atuar em equipe e com ações autônomas e a efetividade do seu trabalho está na possibilidade do diálogo com as tecnologias dura e leve-dura, por meio de seu domínio de tecnologias leve ${ }^{(15)}$.

Sendo assim, a participação do enfermeiro no processo gestacional traz qualidade à assistência de gestantes, uma vez que o objetivo da consulta de pré-natal, instituída pelo Ministério da Saúde ${ }^{(16)}$, tem suas características envoltas para o processo de acolhimento e humanização e, mesmo sendo imprescindível o acompanhamento da gestação de risco pelo profissional médico, isso não exclui a participação de outros profissionais. Em contrapartida, no modelo de atenção médicocentrado, o ambiente que deveria ser de escuta ativa para atender às necessidades biopsicossociais das gestantes de risco, passa a ser uma consulta voltada apenas para o problema clínico sem avaliar as demais condições humanas ${ }^{(14)}$.

Na realização do estudo, percebemos que o enfermeiro prioriza apenas o acolhimento e triagem das gestantes antes de cada consulta prénatal, realizada pelo médico da instituição, dificultando a percepção da contribuição e influência positiva que o profissional de enfermagem tem sobre a assistência pré-natal prestada. Tal pratica contraria a ideia apresentada por Melo et al. ${ }^{(17)}$ de que a consulta de enfermagem garanta maior qualidade da assistência pré-natal, uma vez que o profissional poderá buscar informações mais aprofundadas acerca da experiência gestacional da paciente, permitindo realizar a sistematização de enfermagem e planejar o cuidado de acordo com suas particularidades. A realização das consultas também poderá ser utilizada para embasamento de novas práticas e condutas multiprofissionais na assistência pré-natal.

\section{Representações relacionadas ao medo}

Ao serem questionadas sobre os sentimentos em relação ao risco gestacional, as participantes relacionaram o diagnóstico a hábitos de vida, doenças e complicações desenvolvidas antes ou durante a gestação, demonstrando que isso ocasiona o medo, preocupação com o desenvolvimento e aceitação dessa gestação. As participantes, deste estudo, apresentaram em suas falas o medo de complicações na gestação e no momento do parto. $O$ medo estava relacionado à insegurança no desenvolvimento da gestação e a desfechos negativos no momento do parto. Tais sentimentos são representados nas falas "Eu queria muito ter parto normal, não queria evoluir para uma cesárea. Só que aí envolve múltiplos fatores, eu tive descolamento, né? Então eu não sei se vai dar certo!" (E3). "Então, eu tô com um pouquinho de medo, né? Até mesmo do parto. Por causa da questão da minha pressão, a gente não sabe o que pode acontecer" (E6). "Eu fico com medo que, como eu já tive eclampsia, a minha 
irmã ficou com medo de acontecer (aumento da pressão arterial) na hora do parto" (E10).

Ao analisar os dados coletados, percebemos que a falta de informação e esclarecimentos sobre os riscos, em conjunto com as representações sociais são os principais responsáveis por gerar ansiedade e medo nas participantes em relação ao futuro da gestação e possíveis complicações na saúde do bebê. Almeida et al. ${ }^{(17),}$ em seu estudo realizado com 18 gestantes de risco habitual, observaram que $43 \%$ das participantes do seu estudo consideravam-se não informadas sobre o parto e $34 \%$ possuíam informações incompletas. Esse déficit nas informações influenciou sentimentos negativos e ansiedade nessas participantes que relataram medo de sofrer violência obstétrica, medo de não suportar a dor e o mal- estar do trabalho de parto, medo de morrer, durante o processo de parturição e medo de ser submetida à cesárea.

Cabral et al. ${ }^{(18)}$ trouxeram resultados semelhantes em estudo, realizado com gestantes de alto risco, onde $19 \%$ das entrevistadas demonstraram medo em relação ao desfecho da gestação, a própria saúde e a do bebê e $34 \%$ relataram falta de conhecimento sobre 0 diagnóstico de risco e como isso pode influenciar no desenvolvimento da gestação. Em outro estudo, Vieira et al. ${ }^{(19)}$ apontam que, independentemente da situação de risco ter sido diagnosticada, previamente, ou durante a gestação atual, observaram-se sentimentos de estresse e ansiedade na gestante e em seus familiares.

Com base nos relatos das participantes destes e de outros estudos, é possível perceber que o diagnóstico de risco pode acarretar fatores psíquicos negativos que influenciem, diretamente, o progresso da gestação e a experiência da gestante. Bezerra ${ }^{(20)}$ percebe, em seu estudo, que as medidas de enfrentamento minimizam esses fatores, evidenciando, então, a necessidade de uma pratica de pré-natal com métodos de enfrentamento voltados para a oferta de informações claras sobre o diagnóstico e os riscos envolvidos, com atenção multiprofissional e maior inclusão da família, durante o processo, proporcionando o desenvolvimento de uma experiência positiva para a gestante e seu círculo social.

Mesmo com o sentimento do medo, vale ressaltar que a fé foi citada pelas entrevistadas como um recurso de enfrentamento utilizado para a manutenção da esperança de que a gestação evolua da melhor forma possível. "E Deus há de abençoar porque é só um estágio e eu tenho fé em Deus que logo vai passar. Ai com relação a ter o bebê é igual eu falei inseguranças eu tenho, mas também tenho fé em Deus" (E9).

Porto, ${ }^{(21)}$ ao realizar seu estudo, percebeu que $85 \%$ das gestantes de risco entrevistadas relatavam pertencer a alguma religião e usar a fé para renovação de forças no enfrentamento e aceitação do quadro de risco. A religião tem relação estreita com o estilo de vida, necessitando ser compreendida como algo que dá sentido à situação de crise e sofrimento da usuária e auxilia na recuperação da saúde. Sendo assim, deve ser incentivada pelos profissionais de saúde como peça fundamental para enfrentar o diagnóstico e criar mecanismos que facilitem a assistência prestada e a adesão aos cuidados necessários ao tratamento.

\section{Representações relacionadas à morte}

Quando se questiona sobre o que é gestação de risco, do ponto de vista da gestante, muitas relatam o risco da morte do feto ou da própria morte. "Ali você está com o pé na cova e outro na terra né, então qualquer coisinha pode ser fatal na vida da gente. Meu medo mesmo desde o começo foi do neném morrer ou nascer com alguma sequela" (E9). "Eles falam que pode dar eclâmpsia e pode levar à morte e ai a gente fica apreensivo com isso" (E10). "Uma dificuldade muito grande que uma mulher grávida tem é o risco de perder o bebê. Pra ela é um sofrimento muito grande" (E14).

O medo da perda e o sentimento de impotência fazem com que elas se empenhem no tratamento, a fim de amenizar a situação, principalmente psíquica, fazendo tudo que for possível para impedir que a evolução do parto seja trágica. "Mas com o acompanhamento que a gente tem aqui no alto risco as coisas vão se acalmando, a gente também vai entendendo mais nossa condição e fazendo um acompanhamento correto, um tratamento correto com a medicação" (E8).

Apesar da diminuição considerável das mortes maternas, ${ }^{(1)}$ existem ainda preocupações relacionadas à mortalidade materno-infantil. Em um estudo sobre o perfil epidemiológico de mortalidade materna, é descrito que $95 \%$ dos óbitos maternos são por causas evitáveis, principalmente pela garantia de uma atenção obstétrica de qualidade, abrangendo todas as 
necessidades e demandas vivenciadas pelas gestantes, seja de risco habitual ou alto risco ${ }^{(16)}$.

Corroborando com esses dados, estudo com gestantes portadoras de cardiopatias também apontou a angústia e o medo da morte e de perdas fetais, além do sentimento de vulnerabilidade vivenciado por gestantes em situação de risco ${ }^{(12)}$. Sabe-se que, em todas as gestações, é realizada a classificação de risco para que sejam tomadas as devidas condutas em cada caso. Sendo assim, com a quebra de todo esse processo construído, se acaso venha a existir uma próxima gestação e esta seja classificada de risco, a gestante que teve sua esperança dissipada entenderá que isso pode se repetir e aumentar o risco da tão temida perda da própria vida ou da morte fetal.

Portanto, para que sejam amenizadas as representações relacionadas ao medo da morte, são necessários investimentos nas redes de atenção à saúde, principalmente no processo de referência e contra referência e capacitação dos profissionais que prestam atendimento a essa população, tanto para melhorar o cuidado ao binômio mãe-filho, como para garantir uma assistência mais humanizada e com prestação de informações e esclarecimentos que possibilitarão melhorias no autocuidado, como a redução da ansiedade, do medo e demais aspectos psicológicos, por falta de um manejo multiprofissional.

\section{CONCLUSÃO}

Evidenciou-se que as representações das gestantes de alto risco, giraram em torno de sentimentos de medo relacionados à gestação e à morte, em decorrência dos agravos da condição de risco, além da valorização do modelo biomédico e do profissional de medicina, em detrimento do trabalho em equipe multiprofissional e da promoção da saúde.

Tais sentimentos podem ser trabalhados no acompanhamento multiprofissional, durante a gestação, e o profissional enfermeiro tem um papel primordial como membro da equipe de saúde, podendo identificar as demandas de cada gestante e oferecer um cuidado individualizado, incluindo o suporte emocional e a educação para o autocuidado. Entretanto, é necessário que esse profissional seja capacitado para a tomada de decisões e tenha habilidades de comunicação com a usuária, com outros profissionais e com outros serviços da Rede de Atenção.
Além disso, esse estudo mostrou que há pouco envolvimento da equipe multiprofissional e do profissional enfermeiro nos cuidados diretos com as gestantes de alto-risco, principalmente no que se refere à realização de consultas e orientações individuais e em grupos, o que é condizente com o encontrado em muitos estudos que apontam a valorização dos aspectos biológicos em comparação aos demais aspectos. Visão esta que está intimamente ligada à qualidade da assistência prestada à gestante de alto risco, já que a condição de risco, por si só, eleva os sentimentos negativos sob inúmeros aspectos biopsicossociais. Apesar de abranger as representações sociais de mulheres diagnosticadas com algum risco gestacional, vale salientar as limitações do estudo, uma vez que, o método utilizado não permite a generalização dos resultados.

Contudo, esse estudo poderá contribuir para a ampliação do conhecimento e espaço de discussão sobre gestações de alto-risco, a partir das experiências e representações sociais das gestantes. E, além do mais, poderá facilitar a reflexão dos profissionais para uma assistência humanizada e integral, voltada não apenas para o diagnóstico e condições clínicas, mas também para os aspectos psicossociais. As falhas no atendimento à gestante do serviço especializado podem ser supridas com a organização do fluxo de atendimento e implementação de assistência multiprofissional voltada para as demandas da usuária com o uso de tecnologias leves como consultas de enfermagem e de outros membros da equipe multiprofissional, inclusão na rotina de grupos para trocas de experiência e atendimentos em sala de espera de forma a garantir uma assistência voltada para o acolhimento e escuta qualificada.

\section{REFERÊNCIAS}

1- Ministério da Saúde (BR). Gestação de alto risco: Manual técnico. Brasília: Ministério da Saúde; 2012.

2- Brasil. Portaria no 1459 de 24 de junho de 2011. Institui no âmbito do Sistema Único de Saúde a Rede Cegonha. Diário Oficial da União 2011.

3- Melo MN, Amorim TV, Salimena AMO, Melo MCSC, Souza IEO. Cuidado hospitalar de mulheres que vivenciaram a gestação de risco: Contribuições para a enfermagem. Rev Enferm UFPE 2016;10(11):3911-7. DOI: 10.5205/reuol.9881-87554-1-EDSM1011201612 
4- Riegert IT, Correia MB, Andrade ARL, Rocha FNPS, Lopes LGF, Viana APAL, et al. Avaliação da satisfação de puérperas em relação ao parto. Rev Enferm UFPE 2018;12(11):2986-93. DOI: 10.5205/1981-8963-v12i11a236863p2986-29932018

5- Oliveira DC, Mandú ENT. Women with high-risk pregnancy: Experiences and perceptions of needs and care. Esc Anna Nery 2015;19(1):93-101. DOI: 10.5935/1414-8145.20150013

6- Jardim MJA, Silva AA, Fonseca LMB. The nurse's contributions in prenatal care towards achieving the pregnant women empowerment. J Res Fundam Care 2019;11(2):432-40. DOI: 10.9789/2175-5361.rpcfo.v11.6370

7- Oliveira JCS, Fermino BPD, Conceição EPM, Navarro JP. Assistência pré-natal realizada por enfermeiros: $\mathrm{O}$ olhar da puérpera. $\mathrm{R}$ Enferm Cent O Min. 2015; 5(2):1613-1628. DOI: 10.19175/recom.v0i0.857

8- Almeida AMO, Santos MFS, Trindade ZA. Teoria das Representações Sociais 50 anos. 2a ed. Brasília: Technopolitik; 2014.

9- Nascimento LCN, Souza TV, Oliveira ICS, Moraes JRMM, Aguiar RCB, Silva LF. Saturação teórica em pesquisa qualitativa: Relato de experiência na entrevista com escolares. Rev Bras Enferm. 2018;71(1):228-33. 10.1590/0034-7167-20160616

10- Demazière $D$, Dubar $C$. Analyser les entretiens biographiques: L'exemple de récits d'insertion. Paris: Nathan; 1997.

11- Blanchet A, Gotman A. L'enquête et sés methodes: I'entretien. Paris: Nathan; 1992.

12- Amorim TV, Souza IEO, Salimena AMO, Queiroz ABA, Moura MAV, Melo MCSC. Reproductive risk in pregnant women with heart diseases: The living world guiding health care. Texto Contexto Enferm. 2018;27(2):1-9. 10.1590/0104-070720180003860016

13- Soares LG, Higarashi, IH. Case management as a high-risk prenatal care strategy. Rev Bras Enferm. 2019;72(3): 692-9. DOI: 10.1590/00347167-2018-0483

14- Cardelli AAM, Marrero TL, Ferrari RAP, Martins JT, Serafin D. Expectations and satisfaction of pregnant women: Unveiling prenatal care in primary care. Invest Educ Enferm. 2016; 34(2): 252-260. DOI: 10.17533/udea.iee.v34n2a04 15- Errico LSP, Bicalho PG, Oliveira TCFL, Martins $\mathrm{EF}$. The work of nurses in high-risk prenatal care from the perspective of basic human needs. Rev Bras Enferm. 2018;71(Suppl 3):1257-64. DOI: 10.1590/0034-7167-2017-0328
16- Martins ACS, Silva LS. Epidemiological profile of maternal mortality. Rev Bras Enferm. 2018;71(Suppl 1):677-83. DOI: 10.1590/00347167-2017-0624

17- Melo DEB, Silva SPC, Matos KKC, Martins VHS. Prenatal nursing consultation: social representations of pregnant women. Rev Enferm UFSM 2020;10(18):1-18. DOI: 10.5902/2179769237235

18-Almeida ARV, Alcantara DS, Araujo TT. Expectativas da gestante em relação ao parto. Rev Interdiscip. 2018; 11(1):12-9. DOI: 10.17648\%2F2317-5079.v11n1.1178

19- Cabral SAAO, Alencar MCB, Carmo LA, Barbosa SES, Barros ACCV, Barros JKB. Receios na gestação de alto-risco: Uma análise da percepção das gestantes no pré-natal. Id on Line Rev Mult Psic. 2018;12(40):151-162. DOI: 10.14295/idonline.v12i40.1051

20- Vieira VCL, Barreto MS, Marquete VF, Souza RR, Fischer MMJB, Marcon SS. Vulnerability of high-risk pregnancy in the perception of pregnant women and their families. Rev RENE 2019; 20:1-9. DOI: 10.15253/2175-6783.20192040207

21- Bezerra JC. Modos de enfrentamento e apego materno-fetal em gestantes de alto risco: Um estudo comparativo [dissertação]. Natal: Centro de Ciências Humanas, Letras e Artes; 2017.

22- Porto MA. Gestantes de alto risco em programa de alta hospitalar qualificada: personalidade, estilo de vida e vivencias [dissertação]. São Jose do Rio Preto: Faculdade de Medicina de São José do Rio Preto; 2018.

Nota: Artigo proveniente de Trabalho de Conclusão de Curso Intitulado: 'Representações Sociais de Gestantes que Frequentam Serviço Especializado em Gestações de Alto Risco', do curso de Enfermagem da Universidade Federal de São João Del-rei.

Recebido em: 11/12/19 Aprovado em: 08/05/20

\section{Endereço de correspondência:}

Walquíria Jesusmara Santos

Universidade Federal de São João del-Rei Rua Sebastião Gonçalves Coelho, 400 35501-296 - Chanadour, Divinópolis, MG, Brasil

E-mail:waljsantos@hotmail.com 